\title{
Minimization of Nanodroplet Activation Time using Focused-Pulses for Droplet-Based Ultrasound Super- Resolution Imaging
}

\author{
Ge Zhang ${ }^{1}$, Sevan Harput ${ }^{1}, 2$, Jiaqi Zhu ${ }^{1}$, Kirsten Christensen-Jeffries ${ }^{3}$, Jemma Brown ${ }^{3}$, Chee Hau Leow ${ }^{1}$, Chris \\ Dunsby $^{4 *}$, Robert J. Eckersley ${ }^{3 *}$, Meng-Xing Tang ${ }^{1 *}$ \\ ${ }^{1}$ Department of Bioengineering, Imperial College London, London, UK, SW7 2AZ, United Kingdom \\ ${ }^{2}$ Division of Electrical and Electronic Engineering, London South Bank University, London, SE1 0AA, UK \\ ${ }^{3}$ Biomedical Engineering Department, School of Biomedical Engineering and Imaging Sciences, Kings College London, London, \\ SE1 7EH, UK \\ ${ }^{4}$ Department of Physics and Centre for Pathology, Imperial College London, London, SW7 2AZ, UK \\ * These authors contributed equally to this work \\ Email: Mengxing.Tang@Imperial.ac.uk
}

\begin{abstract}
One of the crucial challenges in the application of ultrasound super-resolution imaging using microbubble contrast agents to the clinic is the long acquisition time. Recently acoustic wave sparsely activated localization microscopy (AWSALM) was developed to activate, image, and destroy nanodroplets to achieve ultrasound super-resolution imaging. The activation and destruction of nanodroplets using focused pulses in AWSALM can generate new localization signals without relying on flow and shows the potential to achieve faster ultrasound super-resolution. However, the technique requires optimization to achieve good activation efficiency with the minimum amount of activation time.
\end{abstract}

This work investigates how the different activation strategies affect the activation time and efficiency. A range of activation sequences with different $F$-numbers and spacing between transmit foci are used and their activation efficiency and the data acquisition time are quantified. The results show that sweeping the focus of the activation pulse with an F-number of 0.4 and a step size of 2.5 wavelengths can generate the highest droplet activation. By using a larger or smaller spacing between consecutive focused activation beams generated lower contrast, which suggests that densely spaced activation pulses may destroy the activated nanodroplets generated by the previous activation pulse. In summary, this study demonstrates that the rate of activation of nanodroplets can be increased by optimizing the Fnumber and the sweep step size. This finding can inform the development of droplet-based super-resolution imaging to further minimize the data acquisition time.

\section{Keywords- Super-Resolution, Decafluorobutane Nanodroplets, Focused Activation, Microbubbles}

\section{INTRODUCTION}

Nanodroplets, also called 'phase-change contrast agents', have been widely investigated in the field of medical ultrasound imaging [1-3]. In localization-based superresolution imaging, there are three main advantages of using nanodroplets compared with the conventional microbubble contrast agents. First, the diameter of a nanodroplet is much smaller than a microbubble: previous theoretical work calculated that nanodroplets are 3-6 times smaller than microbubbles [4]. Their smaller size makes it possible for nanodroplets to flow into smaller vessels or extravasate into tumour tissue where microbubbles cannot. Second, the in vivo circulation time of nanodroplets has been shown to be at least 3-5 times longer than that of microbubbles [ [ $]$ ]. This can potentially allow the scanning session to last longer and the nanodroplet dose to be smaller. Third, the nanodroplets can be manipulated/activated by an ultrasound pulse sequence at a specific time or spatial location to become microbubbles to provide contrast signals. This provides more control and flexibility to the real-time scanning, e.g. to provide myocardium enhancement without chamber enhancement.

Low-boiling-point perfluorocarbon nanodroplets have been investigated as a means for achieving ultrasound superresolution imaging through acoustic switching of such agents, which called 'acoustic wave sparsely activated localization microscopy (AWSALM)' [1]. One of the crucial challenges in the application of ultrasound super-resolution imaging using microbubble contrast agents to the clinic is the long acquisition time [ $[\underline{6}, 7]$. Recently reported fast-AWSALM has demonstrated sub-second frame rate in ultrasound superresolution imaging, where nanodroplets were activated, imaged and destroyed using plane-wave pulses on a micro-second time scale [8]. In deep tissue imaging, focused pulses may be required to activate nanodroplets, as plane-wave pulses may not have sufficient pressure for droplet vaporization. Although a higher imaging frame rate has been investigated, the optimum balance between activation time and activation efficiency using focused pulses has not been explored.

In this study, a range of activation sequences with different F-numbers and arrangements of activation pulses are studied to investigate and optimize the activation efficiency of 
nanodroplets for minimizing the data acquisition time for droplet-based ultrasound super-resolution imaging.

\section{METHODS AND EXPERIMENT}

\section{A. Nanodroplet Preparation}

The preparation of the nanodroplet solution has been adapted from previously described methods [9-11]. The lipid shell was generated by dissolving 1,2-dipalmitoyl-sn-glycero3-phosphocholine (DPPC) and 1,2-distearoyl-sn-glycero-3phosphoethanolamine-N-[amino(polyethylene glycol)-2000] (DSPE-PEG-2000) into a solution of phosphate-buffered saline (PBS), propylene glycol, and glycerol. All the lipids described above were purchased from Avanti Polar Lipids, Inc., USA. The lipid solution was added to a glass vial and the headspace of vial was exchanged with octafluoropropane gas (Fluoromed, USA) via an inlet needle along with a vent needle. Mechanical agitation was sufficient to produce the formation of lipid-shell octafluoropropane-core microbubbles. In order to condense microbubbles into nanodroplets, the headspace of the vial was pressurised while the vial immersed in the ice-water bath according to the previously described method [12-15].

\section{B. Experimental Setup}

2 litres of water was maintained at $37{ }^{\circ} \mathrm{C}$ in a water tank where the walls were covered with acoustic absorbers. A L11$4 \mathrm{v}$ transducer equipped with ultrasound research platform (Verasonics Vantage 128, Kirkland, USA) was placed in the tank. A diluted nanodroplet solution was prepared and injected into the water tank to achieve a concentration of $5.5 \times 10^{6}$ droplets $/ \mathrm{ml}$ [16]. The solution was gently stirred before the experiments to avoid sedimentation.

\section{Activation Strategies}

A customised 'Imaging - Activation - Imaging' sequence [17-19] was implemented on an ultrasound research platform (Verasonics Vantage 128, Kirkland, USA) with a L11-4 38$\mathrm{mm}$ linear array probe (ATL, USA). Different activation strategies were investigated using focused beams for a range of F-numbers $(0.4,0.8$, and 1.2) and step sizes (the focused beam was swept through the region with a step size of $10,5,2.5$, and 1.25 wavelengths, a larger step size means fewer activation pulses used and thus a shorter time for activation). Fig 1 shows the patterns of activation focused beams for the different $\mathrm{F}$ numbers.

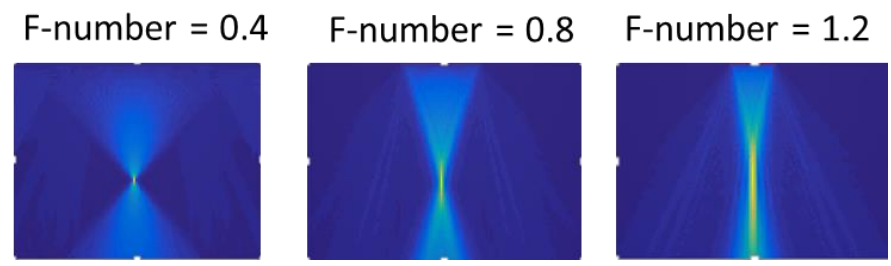

Fig. 1. Three images show the focused beam with F-numbers of $0.4,0.8$ and 1.2 respectively. A focused beam with a larger F-number enables a larger activation area but less activation pressure compared to the focused beam with a smaller F-number using the same voltage input.

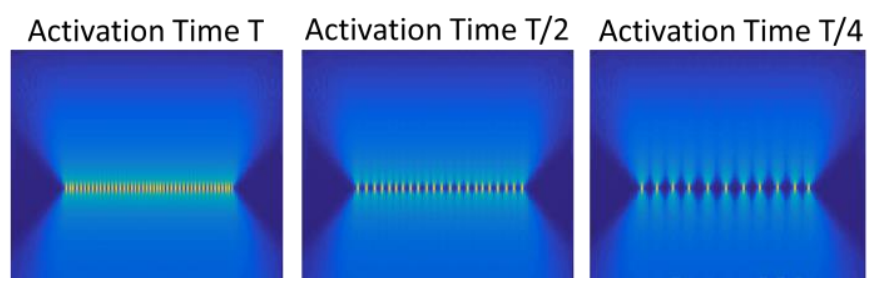

Fig. 2. Three images show the focused beam pattern swept through the region with a step size of $10,5,2.5$, and 1.25 wavelengths respectively. A focused beam with a larger sweeping step size enables a faster activation.

\section{Image Acquisition \& Analysis}

A 1-cycle single-angle plane-wave pulse at $4 \mathrm{MHz}$ with a peak-negative-pressure (PNP) of $0.15 \mathrm{MPa}$ was transmitted in order to image the activated nanodroplets. A 2-cycle focused pulse was transmitted at $4 \mathrm{MHz}$ with a PNP of $4.81 \mathrm{MPa}$ to activate nanodroplets. 100 frames were acquired before and after activation respectively at a frame rate of $1000 \mathrm{~Hz}$. The contrast enhancement (contrast signal after activation subtracts that before activation) was measured for each experiment.

\section{REASULTS AND DISCUSSIONS}

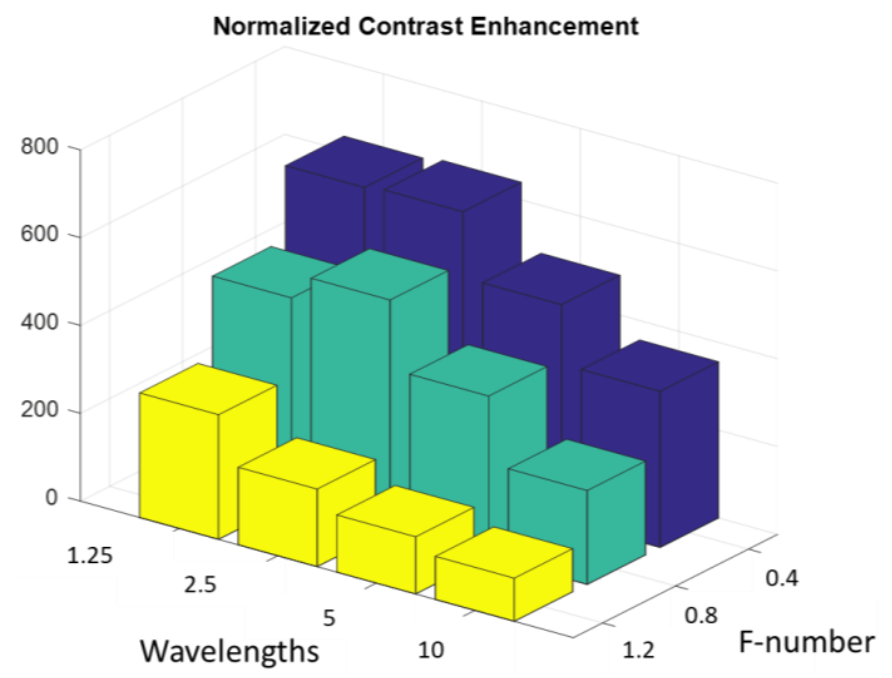

Fig. 3. Normalized contrast enhancement after droplet activation using focused beams with different F-numbers (0.4, 0.8 and 1.2) and step sizes (10, $5,2.5$ and 1.25 wavelengths).

Fig 3 shows the normalized contrast enhancement after droplet activation using focused beams with different F-numbers $(0.4$, 0.8 , and 1.2) and step sizes $(10,5,2.5$, and 1.25). The results show that sweeping the region of interest by advancing the activation pulse with an F-number 0.4 and a step size of 2.5 wavelengths can generate the greatest droplet activation. By advancing the focused activation beam with a step size of 1.25 wavelengths generated lower contrast than using a step size of 2.5 wavelengths, which suggests that neighbouring activation pulse may destroy the activated nanodroplets generated by the previous activation pulse prior to the next imaging pulses. 

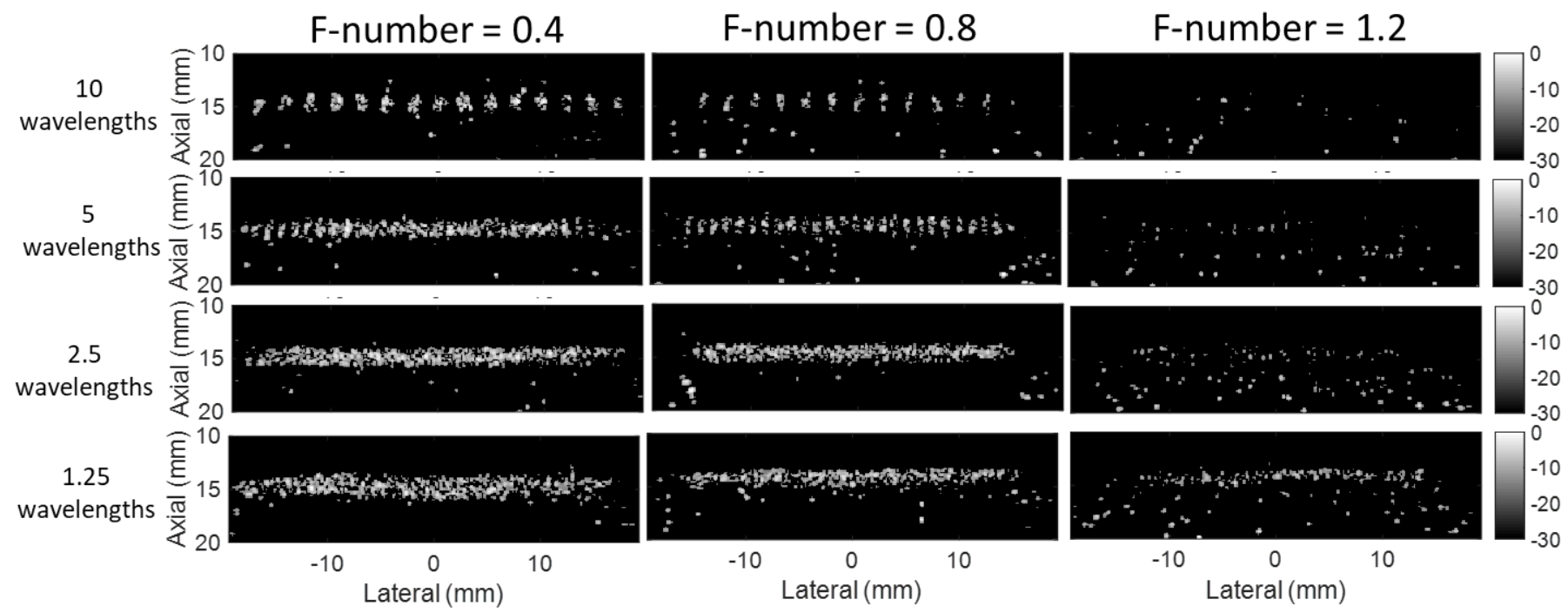

Fig. 4. Representative ultrasound images of activated droplet using focused beams with different F-numbers $(0.4,0.8$ and 1.2$)$ and step sizes $(10,5,2.5$ and 1.25 wavelengths).

Fig 4 shows the representative ultrasound images of activated droplet using focused beams with different F-numbers (0.4, 0.8 and 1.2) and step sizes (10, 5, 2.5 and 1.25 wavelengths). As can be seen from the figure, the activation focused beam with a step size of 2.5 wavelengths can generate a relatively uniform contrast enhancement. An even larger step size (eg. 5 or 10 wavelengths) may be preferred in the ultrasound superresolution scenario because a sparse activation of nanodroplet is desired. This implies the activation time can be even faster (by 8 times compared to the conventional activation pulses). A focused beam with a larger F-number enables a larger activation area but produces a lower activation pressure compared to the focused beam with a smaller F-number using the same voltage input. As suggested by the results, a focused beam with a larger F-number may require a higher voltage input in order to obtain the visible contrast enhancement.

The driving voltage for the transducer is fixed in this study to investigate the impact of F-number and the spacing of the transmit sweeping foci. It would be interesting in the next step to investigate whether increasing the driving voltage closer to the limit would enable even faster activation of the whole field of view by using smaller F-numbers and larger spacing of the foci.

2D matrix-array probe may be employed in the future study to facilitate the acoustic activation of nanodroplets. One of the limitations of using $1 \mathrm{D}$ probe is that only a small population of droplets with the sweeping region could be activated. The application of 2D matrix-array may enable a large activation region by focusing of elements from different rows/columns at different regions. Therefore, the activation efficiency can be potentially significantly increased by using a $2 \mathrm{D}$ matrix-array probe.

\section{CONCLUSION}

This study demonstrates how the different droplet activation strategies affect the activation time and efficiency using focused pulses in AWSALM for fast super-resolution imaging. A range of activation sequences with different Fnumbers and spacing of the sweeping activation pulse foci are studied to investigate and optimize the activation efficiency of nanodroplets for minimizing the data acquisition time. The results show that sweeping the region of interest by advancing the activation pulse with an F-number 0.4 and a step size of 5 wavelengths can generate the most droplet activation. By advancing the focused activation beam with a step size of 1.25 wavelengths generated lower contrast than using a step size of 2.5 wavelengths, which suggests that neighbouring activation pulse may destroy the already activated nanodroplets by the previous activation pulse prior to the next imaging pulses. In summary, this study demonstrates the activation of nanodroplets can be increased by optimizing the F-number and the sweeping step size. This finding can inform the development of droplet-based super-resolution imaging to further minimize the data acquisition time.

\section{ACKNOWLEDGMENT}

This work was supported by the EPSRC under Grant EP/N015487/1 and EP/N014855/1, in part by the King's College London (KCL) and Imperial College London EPSRC Centre for Doctoral Training in Medical Imaging (EP/L015226/1), in part by the Wellcome EPSRC Centre for Medical Engineering at KCL (WT 203148/Z/16/Z), in part by the Department of Health through the National Institute for Health Research comprehensive Biomedical Research Center Award to Guy's and St Thomas' NHS Foundation Trust in 
partnership with KCL and King's College Hospital NHS Foundation Trust, in part by the Graham-Dixon Foundation and in part by NVIDIA GPU grant. This work is also supported by the CRUK Multidisciplinary Project Award (No. C53470/A22353), and Imperial-ICR CRCE pump-priming awards.

\section{REFERENCES}

1. Zhang, G., et al., Acoustic wave sparsely activated localization microscopy (AWSALM): Super-resolution ultrasound imaging using acoustic activation and deactivation of nanodroplets. Applied Physics Letters, 2018. 113(1): p. 014101.

2. Zhang, G., et al. Investigation of Nanodroplet Adhesion to Endothelial Cells under Atheroprone Flow Conditions in 2018 IEEE International Ultrasonics Symposium (IUS). 2018.

3. Toulemonde, M., et al. Flow Visualization through Locally Activated Nanodroplets and High Frame Rate Imaging. in 2018 IEEE International Ultrasonics Symposium (IUS). 2018.

4. Sheeran, P.S., et al., Methods of Generating Sub-Micron Phase-Shift Perfluorocarbon Droplets for Applications in Medical Ultrasonography. IEEE Trans Ultrason Ferroelectr Freq Control, 2016.

5. Sheeran, P.S., et al., Contrast-enhanced ultrasound imaging and in vivo circulatory kinetics with low-boiling-point nanoscale phase-change perfluorocarbon agents. Ultrasound Med Biol, 2015. 41(3): p. 814-31.

6. Christensen-Jeffries, K., et al., Poisson Statistical Model of Ultrasound Super-Resolution Imaging Acquisition Time. IEEE Transactions on Ultrasonics, Ferroelectrics, and Frequency Control, 2019: p. 1-1.

7. Zhu, J., et al., 3D Super-Resolution US Imaging of Rabbit Lymph Node Vasculature in Vivo by Using Microbubbles. Radiology, 2019. 291(3): p. 642-650.

8. Zhang, G., et al., Fast Acoustic Wave Sparsely Activated Localization Microscopy: Ultrasound Super-Resolution Using Plane-Wave Activation of Nanodroplets. IEEE Transactions on Ultrasonics, Ferroelectrics, and Frequency Control, 2019. 66(6): p. 1039-1046.

9. Lin, S., et al., Imaging of vaporised sub-micron phase change contrast agents with high frame rate ultrasound and optics. Physics in Medicine and Biology, 2018.

10. Lin, S., et al., Effects of microchannel confinement on acoustic vaporisation of ultrasound phase change contrast agents. Physics in Medicine \& Biology, 2017. 62(17): p. 6884.

11. Zhang, G., et al. Contrast-Enhanced Photoacoustic Imaging of Lowboiling-point Phase-Change Nanodroplets. in 2019 IEEE International Ultrasonics Symposium (IUS). 2019.

12. Zhang, G., et al. Acoustic response of targeted nanodroplets postactivation using high frame rate imaging. in 2017 IEEE International Ultrasonics Symposium (IUS). 2017.

13. Zhang, G., et al. Photoacoustic Super-Resolution Imaging using Laser Activation of Low-Boiling-Point Dye-Coated Nanodroplets in vitro and in vivo. in 2019 IEEE International Ultrasonics Symposium (IUS). 2019.

14. Zhang, G., et al. Acoustic Wave Sparsely-Activated Localization Microscopy (AWSALM): In Vivo Fast Ultrasound Super-Resolution Imaging using Nanodroplets. in 2019 IEEE International Ultrasonics Symposium (IUS). 2019.

15. Zhang, G., et al. High Signal-to-Noise Ratio Contrast-Enhanced Photoacoustic Imaging using Acoustic Sub-Aperture Processing and Spatiotemporal Filtering. in 2019 IEEE International Ultrasonics Symposium (IUS). 2019.

16. Zhang, G., et al., Quantification of Vaporised Targeted Nanodroplets Using High-Frame-Rate Ultrasound and Optics. Ultrasound in Medicine \& Biology, 2019. 45(5): p. 1131-1142.

17. Lin, S., et al. Vaporising phase change ultrasound contrast agent in microvascular confinement. in 2016 IEEE International Ultrasonics Symposium (IUS). 2016.

18. Zhang, G., et al. Super-Localisation Ultrasound Imaging using Sparse Activation of Low-Boiling-Point Nanodroplets. in The 23rd European Symposium on Ultrasound Contrast Imaging. 2018. Rotterdam, Netherlands.

19. Lin, S., et al. High frame rate ultrasound imaging of vaporised phase change contrast agents. in 2017 IEEE International Ultrasonics Symposium (IUS). 2017. 